\title{
BEBIDA ADICIONADA CON BROSIMUM ALICASTRUM SW.: UNA ALTERNATIVA PARA REQUERIMIENTOS DIETARIOS ESPECIALES.
} DRINK ADDED WITH BROSIMUM ALICASTRUM SW.: AN ALTERNATIVE FOR SPECIAL DIETARY REQUIREMENTS.

Martínez-Ruiz Nina del Rocío ${ }^{1}$, Torres Luis Enrique Javier ${ }^{1}$, del Hierro-Ochoa Julio César ${ }^{1}$ y LarquéSaavedra Alfonso ${ }^{2}$

1 Instituto de Ciencias Biomédicas, Universidad Autónoma de Ciudad Juárez. 2 Centro de Investigación Científica de Yucatán, México.

Citation: Martínez-Ruiz N.R., Torres L.E.J., del Hierro-Ochoa J.C., Larqué-S.A. (2019) Bebida adicionada con Brosimum alicastrum Sw.: una alternativa para requerimientos dietarios especiales. Revista Salud Pública y Nutrición, 18 (3), 1-10.

Editor: Esteban G. Ramos Peña, Dr. CS., Universidad Autónoma de Nuevo León, Facultad de Salud Pública y Nutrición, Monterrey Nuevo León, México. Copyright: (C2019 Martínez Ruiz N.R. et al. This is an open-access article distributed under the terms of Creative Commons Attribution License [CC BY 4.0], which permits unrestricted use, distribution, and reproduction in any medium, provided the original author and source are credited.

Competing interests: The authors have declared that no competing interests exist.

DOI: https://doi.org/10.29105/respyn18.3-1

Recibido: 27 de marzo 2019; $\quad$ Aceptado: 11 de septiembre 2019

Email: nmartine@uacj.mx 


\title{
BEBIDA ADICIONADA CON BROSIMUM ALICASTRUM SW.: UNA ALTERNATIVA PARA REQUERIMIENTOS DIETARIOS ESPECIALES.
}

\author{
Martínez- Ruiz Nina del Rocío ${ }^{1}$, Torres Luis Enrique Javier ${ }^{1}$, del Hierro-Ochoa Julio César ${ }^{1}$ y Larqué-Saavedra \\ Alfonso'. \\ 1 Instituto de Ciencias Biomédicas, Universidad Autónoma de Ciudad Juárez. 2 Centro de Investigación Científica de \\ Yucatán, México.
}

\begin{abstract}
RESUMEN
Introducción. Brosimum alicastrum Sw. (ramón) es un árbol de Mesoamérica y el Caribe, cuya semilla es subutilizada, a pesar de ser un alimento preciado por civilizaciones prehispánicas como los Mayas. La semilla de ramón tiene propiedades de interés por su contenido en macro y micronutrientes. Objetivo: Formular y elaborar una bebida adicionada con harina de semilla de ramón para regímenes dietarios especiales. Material y Método: Se diseñó y formuló una bebida, la cual se analizó en sus propiedades fisicoquímicas ( $A O A C$ ), inocuidad (cuenta directa en placa), aceptación sensorial (escala hedónica de 9categorías) y en respuesta glicémica (curva de tolerancia a la glucosa). Resultados: Se obtuvo una bebida sabor café capuchino, con un contenido de proteína de $6.8 \mathrm{~g}$, fibra dietética de $2 \mathrm{~g}$ y bajo aporte de grasa $(0.2 \mathrm{~g})$, en una porción de $281 \mathrm{ml}$, fue aceptada por el consumidor (75\%) y su ingesta no alteró la respuesta glicémica en individuos jóvenes. Conclusiones: Se obtuvo una bebida de ramón sensorialmente aceptada, que aporta proteína, fibra dietética, es libre de gluten, lactosa y cafeína y su consumo mostró una respuesta normoglicémica, por lo que representa una alternativa para la dieta de jóvenes adultos con intolerancia a lactosa, gluten y/o cafeína.
\end{abstract}

Palabras Clave: Brosimum alicastrum, ramón, bebida nutritiva, alimentación especial.

\section{ABSTRACT}

Introduction: Brosimum alicastrum Sw. (ramón) is a tree from Mesoamerica and the Caribbean, whose seed is currently underutilized, despite being an important food by pre-Hispanic civilizations such as the Mayans. Ramón seed has interesting properties due to its macro and micronutrients. Objective: to formulate and elaborate a nutritive beverage added with ramón seed flour for special feeding regimes. Material and method: A beverage was designed and formulated, which was analyzed in in physicochemical properties (AOAC), innocuity (direct plate count), sensory acceptance (9-point hedonic scale) and glycemic response (glucose tolerance curve). Results: A beverage cappuccino-coffee flavor, was obtained, with a protein content of $6.8 \mathrm{~g}, 2 \mathrm{~g}$ dietary fiber and low-fat intake $(0.2 \mathrm{~g})$, in a portion of $281 \mathrm{ml}$. The product was accepted by the consumer $(75 \%)$ and its intake did not alter the glycemic response in young individuals. Conclusions: A ramón beverage was obtained. It was sensorially accepted provides protein, dietary fiber, is gluten-free, lactose-free and caffeine-free. The beverage was sensorially accepted and its consumption showed a normoglycemic response, so ramón beverage represents an alternative for the diet of young adults with lactose, gluten and / or caffeine intolerance.

Key words: Brosimum alicastrum, ramón, nutritive beverage, special food. 


\section{Introducción}

Ciertos grupos de la población están sujetos a requerimientos dietéticos especiales debido a la intolerancia, hipersensibilidad o patología que presentan y cuyo tratamiento más efectivo se restringe a terapia nutricional. En el caso de los celíacos, su alimentación se restringe a productos libres de gluten, excluyendo cereales como el trigo, centeno, cebada y alimentos que los contengan, sin embargo, generalmente los alimentos libres de gluten no se enriquecen o fortifican por lo que estos productos no aportan la misma cantidad de nutrientes que sus homólogos con gluten (Molina-Rosell, 2013). El eliminar estos granos de amplio consumo e incluso la avena en la enfermedad celíaca, reduce considerablemente la selección de alimentos y frecuentemente estos pacientes presentan déficit de micronutrientes como hierro, ácido fólico, cobre, zinc, vitaminas, entre otros (Moscoso \& Quera, 2015). Por otra parte, la deficiencia de lactasa ocurre en el $70 \%$ de la población adulta en el mundo, particularmente su producción intestinal declina a partir de los dos años, adolescentes y adultos tienen sólo un 5 a $10 \%$ de la concentración de lactasa que existía en la infancia, además de los casos de deficiencia primaria o por enfermedades que afectan la concentración de esta enzima en el intestino (Rosado, 2016). El tratamiento en el 70-80\% de los casos es una dieta sin lactosa o en su caso, mantener una ingesta reducida de leche y sus derivados (Moreira \& López San Román, 2006). Otro grupo con requerimiento dietético especial son las personas sensibles a la cafeína, ya pueden presentar síntomas negativos después de su consumo. En adolescentes se ha observado particularmente episodios depresivos, estrés y ansiedad (Richards \& Smith, 2015) y/o efectos hipertensivos, disturbios del sueño, insomnio, entre otros (Yang, Palmer, \& de Wit, 2010). Estos requerimientos especiales de alimentación suelen ser costosos en algunos casos y difíciles de implementar en la vida diaria. La terapia nutricional requiere del desarrollo de alimentos nutritivos, sensorialmente aceptados por el consumidor y que apoyen la alimentación de este tipo de grupos.

Por otra parte, Brosimum alicastrum Sw. es un árbol neotrópico que se distribuye desde de México, América Central hasta el norte de América del Sur y ha sido utilizado históricamente como alimento desde el período clásico de los Mayas. "Brosimos" significa "comestible" y tiene más de 50 nombres comunes, tales como: ramón, nuez Maya, mojo, mojote, ojite, capomo, huje, ox, entre otros (Barrance et al., 2003). De acuerdo con la Organización de las Naciones Unidas para la Alimentación y la Agricultura (FAO, por sus siglas en inglés), esta especie está clasificada como "Grass", es decir inocua y ha sido utilizada, convirtiendo el fruto, la semilla, madera, hojas y látex, en fuentes de materias primas para alimentación y forrajes (CONAFORFAO, 2012). La Academia de Ciencias de los Estados Unidos lo considera como una de las especies subexplotadas con promisorio valor económico (NAS, 1975). Las semillas de Brosimum alicastrum $\mathrm{Sw}$. presentan un perfil nutrimental alto, de mayor calidad que el maíz (Peters \& PardoTejeda, 1982). Esta semilla tiene propiedades de interés como son su contenido en proteína (13\%), fibra dietética (15\%), bajo contenido de grasa (1.1\%) y un importante aporte en micronutrientes como ácido fólico, riboflavina, vitamina B6, vitamina C, vitamina $\mathrm{A}$, cobre, hierro, magnesio, fósforo, potasio y zinc (USDA, 2017; Carter, 2015; Larqué-Saavedra, 2014a), los cuales son mayores a los que aporta una harina integral de maíz amarillo (USDA, 2017). En sus propiedades antioxidantes, la harina de semilla de B. alicastrum Sw. contiene más compuestos polifenólicos que la nuez, el cacahuate o la almendra y una capacidad antioxidante similar a la nuez, pero mayor que la del cacahuate y la almendra (Tokpunar, 2010; Ozer, 2017). Estas características nutrimentales y en compuestos bioactivos hace de la semilla de Brosimum alicastrum Sw. una propuesta de innovación forestal de la biodiversidad que puede ser aprovechada en el diseño de alimentos nutritivos.

En México la harina de semilla de ramón es un producto orgánico, sin gluten (Larqué-Saavedra, 2014c), su aporte en macro y micronutrientes resulta particularmente importante para la dieta por enfermedad celíaca. Además, la harina de semilla de ramón no aporta lactosa y favorece la elaboración de bebidas de apariencia láctea, lo que puede satisfacer las expectativas del consumidor intolerante a la lactosa. Finalmente, la semilla de ramón no contiene cafeína, pero su semilla tostada se ha utilizado como sustituto de café (Gutiérrez, 2017). Por lo que el objetivo del presente estudio fue formular y elaborar una bebida nutritiva adicionada con harina de semilla de ramón, sensorialmente agradable para apoyar la alimentación especial de ciertos grupos de jóvenes 
adultos como los celíacos, intolerantes a la lactosa y/o sensibles a la cafeína.

\section{Material y Método}

Diseño de la formulación y elaboración de la bebida Para la formulación inicial de la bebida se utilizó como materia prima: harina de semilla de Brosimum alicastrum Sw. (Herbario Roger Orellana s/n, Centro de Investigación Científica de Yucatán (CICY)). La semilla se recolectó de árboles de ramón que crecen en el Rancho Xoccheila (20o33'N;89o34'W), municipio de Sacalum, Yucatán; posteriormente se secó al sol, se removieron las testas y se realizó la molienda hasta obtener una harina fina (MartínezRuiz y Larqué-Saavedra, 2018) (materia prima proporcionada por el CICY). También se utilizó caseína micelar (Muscle Feast ${ }^{\circledR}$ ), cocoa en polvo (Molina $\left.{ }^{\circledR}\right)$, extracto de vainilla (Molina $\left.{ }^{\circledR}\right)$, edulcorante no calórico (sucralosa, Splenda ${ }^{\circledR}$ ), leche descremada y deslactosada (Lala ${ }^{\circledR}$ ) agua purificada (Ciel®), jugo de naranja (Jumex®) y sabor capuchino, moka y chocolate (Deiman $\left.{ }^{\circledR}\right)$. La formulación inicial se propuso en función de la formulación empleada para hacer un atole con la harina de semilla de ramón $(70$ g/ $300 \mathrm{~mL}$ agua $)$ (Arévalo \& Bressani, 2013), la cual se consideró en un 50\% y en una porción de $281 \mathrm{~mL}$ (33 g de harina de semilla de ramón / $281 \mathrm{~mL}$ ). Para la elaboración inicial de la bebida se siguió el método reportado por Acosta y Martínez-Ruiz, (2017) con modificaciones.

Para lo cual realizó homogenizando en un procesador de alimentos (Oster $\left.{ }^{\circledR}\right) 11.7 \mathrm{~g}$ de la harina de semilla de Brosimum alicastrum Sw., junto con $0.85 \mathrm{mg}$ de sucralosa, $0.6 \mathrm{~g}$ de cocoa en polvo y $100 \mathrm{~mL}$ de agua purificada. La mezcla se homogenizó por aproximadamente $2 \mathrm{~min}$ a velocidad baja. Posteriormente se agregó caseína micelar (1-2 g) hasta lograr la consistencia adecuada de la bebida y se homogenizó por $2 \mathrm{~min}$. La bebida obtenida se llevó a un proceso térmico de $83{ }^{\circ} \mathrm{C} / 2 \mathrm{~min}$. Inmediatamente el producto se envasó en caliente en frascos de vidrio con tapa hermética y sello de goma, previamente esterilizados $\left(120{ }^{\circ} \mathrm{C} / 20 \mathrm{~min}\right)$. La bebida se dejó enfriar hasta la formación de vacío y se almacenó a temperatura ambiente. Una vez abierta la bebida se mantuvo en refrigeración $\left(4-6{ }^{\circ} \mathrm{C}\right)$. A partir de la formulación y proceso de elaboración inicial de la bebida, se realizaron pruebas preliminares de nivel de agrado con 10 consumidores, quienes evaluaron y proporcionaron comentarios en la papeleta de respuesta correspondiente sobre la bebida de cada formulación. Con estos resultados preliminares se fue modificando la formulación y el proceso de elaboración de la bebida nutritiva, se realizaron un total de 22 formulaciones efectuando diferentes combinaciones de los ingredientes y modificando el proceso de elaboración. La selección de la formulación y proceso de elaboración final se consideró cuando las pruebas sensoriales preliminares mostraron el mayor grado de aceptación, lo cual se validó posteriormente con una prueba de consumidor.

Análisis fisicoquímico de harina de semilla de ramón y la bebida final

Se realizó un análisis proximal de la bebida obtenida a partir de la formulación y proceso de elaboración final (formulación 22) que incluyó: contenido de humedad (AOAC 935.29), cenizas (AOAC 945.46), proteína (AOAC 984.13), grasa (AOAC 920.39) y carbohidratos totales por el método de diferencia (AOAC, 2000). Además, se realizó la determinación de fibra cruda por una digestión ácido-alcalina (NMX-F-090-S-1978) (SSA, 1979), actividad de agua (AquaLab®, Serie 3), pH por método potenciométrico (NMX-F-317-S-1978) (SSA, 1978), y contenido de sodio por el método de Volhard (NMX-F-360-S-1981) (SSA, 1981). La estimación de fibra dietética se obtuvo multiplicando el porcentaje de fibra cruda, determinado en este estudio, por 3.81. Este valor se calculó mediante la relación del porcentaje de fibra dietética reportado para la harina de semilla de ramón por LarquéSaavedra (2014a) entre el porcentaje de fibra cruda obtenido por Arévalo y Bressani (2013).

\section{Calidad microbiológica de la bebida final}

Se realizó el recuento total de mesofílicos aerobios, coliformes totales y hongos y levaduras por el método de cuenta directa en placa, utilizando placas $3 \mathrm{M}{ }^{\circledR}$ Petrifilm y de acuerdo con el método especificado por el fabricante (México 3M, 2017).

\section{Prueba de consumidor}

La prueba se realizó en 142 consumidores jóvenes universitarios (21.5 \pm 5.3 años). Para la prueba se presentó a cada consumidor $10 \mathrm{~mL}$ de la bebida contenidos en vasos plásticos $(1 \mathrm{oz})$, identificados con números aleatorios de tres dígitos y de manera monádica. La bebida se sirvió a temperatura ambiente y al consumidor se le presentó una escala 
hedónica con nueve categorías desde "Me gusta muchísimo" hasta me "Me disgusta muchísimo". Antes de probar la bebida, se les solicitó a los participantes enjaguarse la boca con agua purificada y posteriormente registrar en la escala hedónica el nivel en que les agrado el producto (Lawless \& Heymann, 2010).

\section{Respuesta glicémica}

Para la prueba de respuesta glicémica se siguió la metodología propuesta por Arévalo y Bressani (2013) con modificaciones. Para lo cual en la prueba participaron 12 jóvenes universitarios cuyos criterios de selección fueron no tener ningún padecimiento crónico, no estar bajo tratamiento farmacológico y que firmaran un consentimiento informado. El ensayo se realizó en dos sesiones, con tres días de diferencia, con el mismo grupo de participantes y un ayuno previo a las pruebas de $8 \mathrm{~h}$. Se elaboraron dos preparaciones de la bebida formulada, una sin harina de semilla de ramón (bebida control, BC) y otra con harina de semilla de ramón (bebida tratamiento, BR) y posteriormente se adicionaron $190 \mathrm{~g}$ de glucosa $\left(70 \%\right.$, Deiman $\left.{ }^{\circledR}\right)$ en $250 \mathrm{ml}$ de cada bebida para obtener una concentración de glucosa de $0.3 \mathrm{~g} / \mathrm{ml}$.

En cada prueba se recolectó al inicio una muestra de sangre por punción venosa. Posteriormente, los participantes ingirieron $125 \mathrm{~mL}$ de la bebida (BC en la primera sesión y BR en la segunda sesión) y 5 min después los $125 \mathrm{~mL}$ restantes. En ambas sesiones, se recolectaron muestras de sangre de los participantes a los 30, 60 y 120 min después del consumo total de la bebida. La concentración de glucosa sérica se determinó por el método enzimático-colorimétrico (COBAS $®$, mod. Integra Plus 400) (Davidsohn \& Bernard, 1983). Todos los procedimientos fueron aprobados por el Comité de Bioética de la Universidad Autónoma de Ciudad Juárez y los participantes firmaron un consentimiento informado.

\section{Análisis de datos}

Los datos provenientes del análisis fisicoquímico se presentan como la media de tres réplicas con desviación estándar, considerando un coeficiente de variación menor al 5\%. Con los datos del análisis proximal se calculó la etiqueta nutrimental del producto y se comparó con productos comerciales similares. Los datos provenientes de la prueba de nivel de agrado se analizaron usando chi cuadrada y ANOVA de una vía. Los datos provenientes de la prueba de tolerancia a la glucosa se analizaron mediante la prueba t-Student. Todas las pruebas se realizaron utilizando el programa XLSTAT, versión 2015 (Addinsoft, París, Francia). En todos los casos se consideró una significancia a $\mathrm{p}<0.05$.

\section{Resultados y Discusión}

Formulación y elaboración de la bebida

Los resultados de las pruebas preliminares de nivel de agrado de las formulaciones previas que permitieron seleccionar la formulación y el proceso de elaboración final de la bebida se muestran en el Tabla I. La formulación 22 (formulación final) presentó las mejores características, y las materias primas utilizadas en su elaboración fueron: harina de semilla de Brosimum alicastrum Sw., cocoa en polvo, extracto de vainilla, edulcorante no calórico (sucralosa), leche descremada y deslactosada, agua y sabor café capuchino (solicitud de patente MX/a/2019/000622), que por su naturaleza la hacen una bebida libre de lactosa, gluten y cafeína $(<1$ $\mathrm{mg} / 100 \mathrm{~mL}$ ).

\begin{tabular}{|c|c|c|}
\hline Formulación & $\begin{array}{l}\text { Observaciones en pruebas sensoriales } \\
\text { preliminares }\end{array}$ & Tipo de modificación \\
\hline F1 inicial & $\begin{array}{l}\text { Muy espesa, sabor amargo, falta dulzor, } \\
\text { arenosa }\end{array}$ & Ingredientes y proceso (homogenizado) \\
\hline $\mathrm{F} 2 \mathrm{yF} 3$ & Ligeramente espesa, poco sabor & $\begin{array}{l}\text { Ingredientes y proceso (1er. tratamiento } \\
\text { térmico) }\end{array}$ \\
\hline F4 & Sabor amargo, falta dulzor & $\begin{array}{l}\text { Ingredientes y proceso (1er. tratamiento } \\
\text { térmico) }\end{array}$ \\
\hline F5 & $\begin{array}{c}\text { Ligero sabor a chocolate, ligeramente } \\
\text { arenoso }\end{array}$ & $\begin{array}{l}\text { Ingredientes y proceso (1er. tratamiento } \\
\text { térmico) }\end{array}$ \\
\hline F6yF7 & $\begin{array}{c}\begin{array}{c}\text { Consistencia agradable, buen aroma, ligero } \\
\text { olor a café, buen dulzor, ligeramente } \\
\text { arenoso y amargo }\end{array} \\
\end{array}$ & $\begin{array}{l}\text { Ingredientes y proceso (prueba de } \\
\text { tamizado) }\end{array}$ \\
\hline F8 & Ligeramente ácida, textura arenosa & Ingredientes \\
\hline F9 y F10 & Buen sabor y dulzor, ligeramente arenoso & Ingredientes \\
\hline F11 & $\begin{array}{l}\text { Sabor a café, buena consistencia, } \\
\text { ligeramente arenoso }\end{array}$ & Proceso (prueba de tamizado) \\
\hline F12 & Muy dulce y ácido, ligeramente arenoso & Ingredientes \\
\hline F13 & $\begin{array}{c}\text { Buen sabor, falta dulzor, olor a café, } \\
\text { ligeramente arenoso }\end{array}$ & $\begin{array}{l}\text { Ingredientes y proceso (2do. tratamiento } \\
\text { térmico, eliminación de tamizado) }\end{array}$ \\
\hline F14 y F15 & Falta de sabor, buena consistencia & Ingredientes \\
\hline F16-F21 & $\begin{array}{c}\text { Sabor agradable, textura ligeramente } \\
\text { cremosa }\end{array}$ & $\begin{array}{l}\text { Ingredientes (se probaron saborizantes: } \\
\text { moka, chocolate y capuchino }\end{array}$ \\
\hline F22 final & $\begin{array}{l}\text { Consistencia agradable, sabor preferido, } \\
\text { dulzor adecuado. }\end{array}$ & \\
\hline
\end{tabular}

El proceso de elaboración de la bebida inicial se fue ajustando a través de las diferentes formulaciones y de acuerdo con el comportamiento de la materia prima, (Figura 1) (solicitud de patente MX/a/2019/000622), indicando los puntos críticos de control y el cual se diseñó considerando etapas de bajo costo para ser implementado con facilidad.

En el proceso se identifican algunos puntos importantes como el primer tratamiento térmico (70 
${ }^{\circ} \mathrm{C} / 2 \mathrm{~min}$ ), el cual tuvo como propósito la gelatinización del almidón contenido en la formulación, pues los gránulos de este no son solubles en agua fría y el calor ocasiona un hinchamiento irreversible del gránulo (Pérez, Baldwin, \& Gallant, 2009). Posteriormente, en los puntos críticos de control destacan: un segundo tratamiento térmico $\left(83^{\circ} \mathrm{C} / 1 \mathrm{~min}\right)$, homogenizado (1 min) y envasado. El objetivo del segundo tratamiento térmico fue eliminar microorganismos patógenos que pueden causar daño a la salud de los consumidores, así como microorganismos deteriorativos o que puedan reducir la vida de anaquel del producto. El tipo de tratamiento empleado para esta bebida, fue tipo una ultrapasteurización a alta temperatura $\left(83^{\circ} \mathrm{C}\right)$ y de tiempo corto (1 $\mathrm{min})$, la cual elimina los microorganismos que están presentes en ella, y por el tiempo de exposición garantiza no afectar los componentes químicos de la misma (Bedolla et al., 2004). El segundo homogenizado permitió romper los gránulos de almidón hinchados por el primer tratamiento térmico y obtener una bebida homogénea de mayor fluidez o consistencia líquida, finalmente el envasado en caliente permitió mantener la inocuidad del producto.

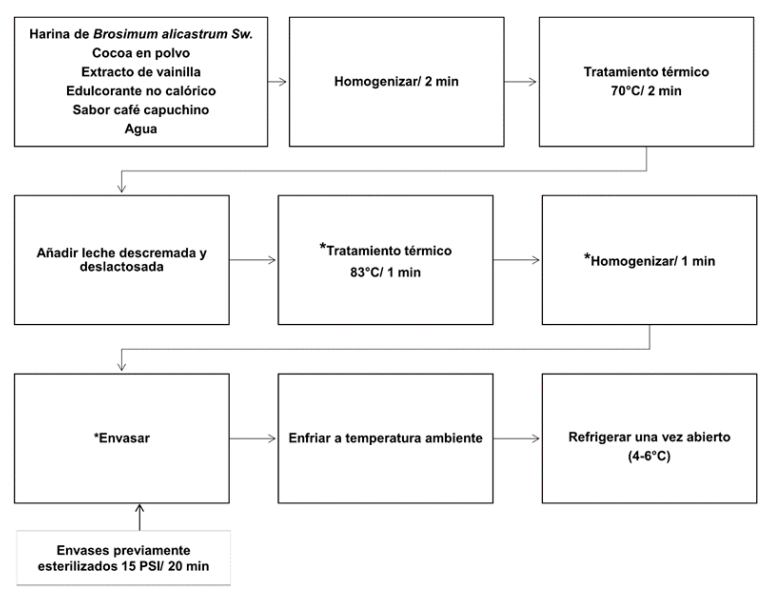

Figura 1. Proceso final para elaboración de la bebida adicionada con harina de semilla de ramón†े. *Puntos Críticos de Control. †Solicitud de patente MX/a/2019/000622.

Composición y aporte nutrimental de la bebida El análisis fisicoquímico de la harina de semilla de ramón y la bebida se presenta en la Tabla II.

\begin{tabular}{|c|c|c|}
\hline & $\begin{array}{c}\text { Harina de semilla de } \\
\text { Brosimum alicastrum Sw. }\end{array}$ & $\begin{array}{l}\text { Bebida con harina de semilla } \\
\text { de Brosimum alicastrum Sw. }\end{array}$ \\
\hline Humedad (\%) & $9.2 \pm 0.2$ & $88.3 \pm 0.0$ \\
\hline Proteína (\%) & $11.1 \pm 0.3$ & $2.4 \pm 0.1$ \\
\hline Grasa (\%) & $0.7 \pm 0.0$ & $0.6 \pm 0.1$ \\
\hline Cenizas (\%) & $3.4 \pm 0.1$ & $0.6 \pm 0.0$ \\
\hline Carbohidratos totales (\%) & $75.6 \pm 0.4$ & $8.5 \pm 0.1$ \\
\hline Fibra cruda (\%) & $4.0 \pm 0.1$ & $0.2 \pm 0.0$ \\
\hline Fibra dietaría $(\%)$ ** & 15.3 & 0.7 \\
\hline $\mathrm{pH}$ & $5.7 \pm 0.0$ & $6.5 \pm 0.6$ \\
\hline Aa & $0.53 \pm 0.0$ & $0.99 \pm 0.0$ \\
\hline Sodio (mg/100g) & & $18.8 \pm 0.0$ \\
\hline
\end{tabular}

La harina de semilla de ramón mostró un alto contenido de proteína, fibra dietética y minerales, así como un bajo contenido de grasa, un $\mathrm{pH}$ ligeramente ácido y una actividad de agua intermedia (zona II). Por su parte la bebida formulada destacó, dentro de sus componentes, en proteína y carbohidratos particularmente complejos, con un $\mathrm{pH}$ ligeramente ácido y una actividad de agua en zona III. El contenido de proteína de la bebida es una mezcla de proteínas de origen animal proporcionadas por la leche $(59 \%)$ y vegetales proporcionadas por la harina de semilla de ramón (41\%). De acuerdo con un estudio anterior, la harina de semilla de ramón proporciona nueve aminoácidos esenciales (Carter, 2015) en mayor cantidad comparado con la leche de vaca (USDA, 2017), lo que la hace una proteína completa. Además, se ha reportado que esta harina proporciona un importante contenido de minerales entre los que destacan cobre $(0.4 \mathrm{mg} / 100 \mathrm{~g})$, hierro $(1.6 \mathrm{mg} / 100 \mathrm{~g})$, magnesio $(53.2 \mathrm{mg} / 100 \mathrm{~g})$, manganeso $(0.7 \mathrm{mg} / 100 \mathrm{~g})$, fósforo $(130.8 \mathrm{mg} / 100$ g), potasio $(431.4 \mathrm{mg} / 100 \mathrm{~g})$, selenio $(31.2 \mathrm{mg} / 100$ g) y zinc $(1.0 \mathrm{mg} / 100 \mathrm{~g})$ en mayor contenido comparada con la leche de vaca. En vitaminas sobresale su aporte en riboflavina $(2.5 \mathrm{mg} / 100 \mathrm{~g})$, ácido pantoténico $(0.8 \mathrm{mg} / 100 \mathrm{~g})$, niacina (1.5 $\mathrm{mg} / 100 \mathrm{~g})$ y timina $(0.2 \mathrm{mg} / 100 \mathrm{~g})$ (USDA, 2017; Carter, 2015). Dicha composición hace de esta harina una materia prima de interés para incrementar la riqueza nutrimental de la bebida. La harina de semilla de ramón proporciona un bajo contenido de grasa, conformada por ácido linoleico $(55.1 \%)$ y linolénico (9.9\%) (Tokpunar, 2010), principalmente, mismos que son ácidos grasos esenciales (Omega 6 y 3, respectivamente) asociados con la salud cardiovascular (Connor, 2000).

De acuerdo con su aporte nutrimental, la bebida formulada en este estudio ("bebida de ramón"), contribuye con el $9 \%$ de proteína $(6.8 \mathrm{~g})$ de la ingesta diaria recomendada (IDR, basada en una dieta de 
$2000 \mathrm{kcal})$, el $0.3 \%$ de grasas $(0.2 \mathrm{~g})$, el $8 \%$ de carbohidratos ( $24 \mathrm{~g}$, de los cuales $8.7 \mathrm{~g}$ son azúcares), el $8 \%$ de fibra dietética $(2.0 \mathrm{~g})$, el $3 \%$ de sodio (63.5 $\mathrm{mg}$ ) y un aporte energético de $125 \mathrm{kcal}$, todo en una porción sugerida de $281 \mathrm{~mL}$. La bebida de ramón aporta menos energía que la leche entera $(175 \mathrm{kcal} /$ $281 \mathrm{~mL}$ ), que un jugo de fruta comercial (132 $\mathrm{kcal} / 281 \mathrm{~mL})$ y similar a una bebida de soya (118 $\mathrm{kcal} / 281 \mathrm{~mL}$ ). La bebida de ramón tiene un importante aporte de proteína en comparación con otras bebidas como la de coco $(0 \mathrm{~g} / 281 \mathrm{~mL})$, almendra $(3.6 \mathrm{~g} / 281 \mathrm{~mL})$ y similar a una bebida de soya sabor chocolate $(6.4 \mathrm{~g} / 281 \mathrm{~mL})$ (USDA, 2017).

El contenido proteico de la bebida de ramón ( $14.5 \mathrm{~g} /$ L) fue ligeramente menor al de un producto lácteo combinado $(15.0 \mathrm{~g} / \mathrm{L})$, lo cual la clasifica como una bebida (NOM-183-SCFI-2012) (SE, 2012). Por otra parte, la bebida de ramón tiene un bajo aporte de grasa en comparación con la leche entera $(9.3 \mathrm{~g} / 281$ $\mathrm{mL})$, bebidas de coco $(5.3 \mathrm{~g} / 281 \mathrm{~mL})$, almendra (2.3 $\mathrm{g} / 281 \mathrm{~mL})$ y de soya $(4.7 \mathrm{~g} / 281 \mathrm{~mL})$. La bebida de ramón no contiene lactosa y su contenido de azúcares, principalmente provenientes de la leche deslactosada (77\%), es similar a una bebida de coco $(8.2 \mathrm{~g} / 281 \mathrm{~mL})$ y menor a la leche entera $(12.8 \mathrm{~g} / 281$ $\mathrm{mL})$, una bebida de soya sabor chocolate $(22.1 \mathrm{~g} / 281$ $\mathrm{mL})$, un jugo de fruta comercial $(30.9 \mathrm{~g} / 281 \mathrm{~mL}) \mathrm{o}$ de un refresco $(29.7 \mathrm{~g} / 281 \mathrm{~mL})$. En fibra dietética, la bebida de ramón aporta dos veces más que bebidas como la de coco $(1 \mathrm{~g} / 281 \mathrm{~mL})$, almendra $(1 \mathrm{~g} / 281$ $\mathrm{mL})$ o un jugo comercial $(0.8 \mathrm{~g} / 281 \mathrm{~mL})$ y una propiedad nutrimental con respecto a la leche entera que no aporta este nutrimento. Finalmente, en sodio la bebida de ramón aporta menor cantidad en comparación con la leche entera $(140.5 \mathrm{mg} / 281 \mathrm{~mL})$, que una bebida de soya $(92.5 \mathrm{mg} / 281 \mathrm{~mL})$ y una de almendra (211 mg/281 mL) (USDA, 2017). Esta información permite considerar a la bebida de ramón como un alimento que aporta calidad y cantidad de proteína y fibra dietética en referencia a bebidas similares; tiene un limitado aporte de lípidos, azúcares y sodio en relación a un alimento completo como la leche entera, o de otros alimentos como jugos comerciales o refrescos; no contiene lactosa, gluten o cafeína en su formulación, lo que la hace una bebida óptima para el desayuno y merienda de jóvenes adultos intolerantes a la lactosa, celíacos o con sensibilidad a la cafeína.

\section{Calidad microbiológica de la bebida}

El resultado de las pruebas microbiológicas mostró < $10 \mathrm{UFC} / \mathrm{mL}$ para coliformes totales, negativo para mesofílicos aerobios y hongos y levaduras. El control en el proceso de elaboración de la bebida de ramón indicó una calidad microbiológica adecuada, haciendo de este producto, un alimento apto para consumo humano (NOM-184-SSA1-2002) (SS, 2002). Resultados similares se obtuvieron en una bebida elaborada a base de arroz y plasma bovino y porcino, con tratamientos térmicos similares a los establecidos en el proceso de elaboración de la bebida de este estudio y que también fueron determinantes para garantizar la inocuidad en el caso de la bebida de arroz (Tirado, Montero, \& Acevedo, 2015). El manejo de puntos críticos de control y su envasado en caliente permitió conservar la bebida a temperatura ambiente, lo cual proporciona un fácil manejo y menor costo de almacenamiento del producto. Una vez abierta la bebida se recomienda su almacenamiento en refrigeración (4-6 $\square$ C). No obstante, más estudios sobre estabilidad y vida de anaquel del producto son necesarios para determinar su fecha de caducidad.

\section{Aceptación de la bebida}

Para evaluar la aceptación de la bebida de ramón, se agruparon las respuestas de la escala hedónica en tres áreas: agrado, neutral y desagrado (Figura 2A). Los resultados indicaron que la bebida fue del agrado del consumidor $(75 \%)$, el desagrado y neutro no presentaron diferencia significativa entre sí $(\chi$ $2=165.8, \mathrm{p}<0.01)$. El consumidor ubicó la bebida en la categoría "Me gusta" y el nivel de desagrado, significativamente menor, se ubicó entre "Me disgusta" y "Me disgusta ligeramente" (Figura 2B) F $(1,128)=299.1, p<0.01$. En función del perfil de macronutrientes y sus características de estar libre de lactosa, gluten y cafeína, la bebida formulada podría considerarse un candidato viable para probar su funcionalidad en diferentes grupos con requerimientos dietarios especiales. Sin embargo, la aceptación del alimento por parte del consumidor es un factor fundamental para que se dé la elección y consumo de este producto sobre otros alimentos que están en el mercado (Suri et al., 2019). Actualmente existen alimentos que se denominan funcionales en el mercado, pero que no son del agrado del consumidor, lo que constituye un fracaso a las propiedades del alimento frente al rechazo del consumidor. Particularmente la FAO recomienda 
considerar alimentos integrados en la cultura de los individuos, con un apropiado aporte de nutrimentos por grupo de edad, enfocados a necesidades especiales y que sean de la preferencia de los individuos (Sundaram, Rawal, \& Clarck, 2015).

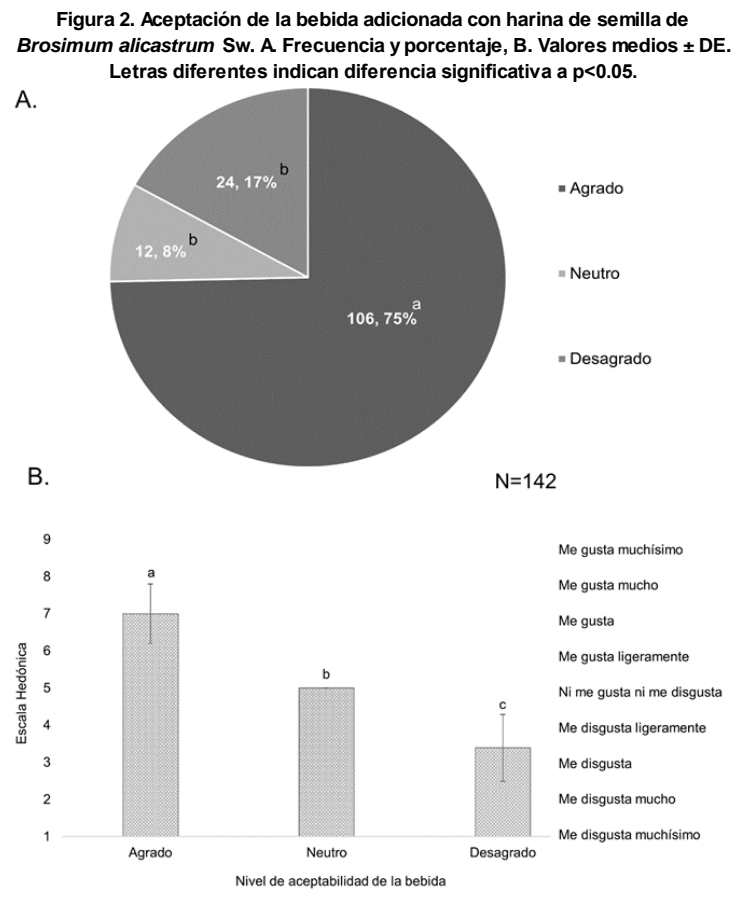

Respuesta glicémica

Los resultados de la prueba se presentan en la Figura 3 . No se encontró diferencia significativa entre BC y BR en la glicemia de los participantes a diferentes tiempos, excepto en la inicial (t-0) donde los participantes con la BC tuvieron una glicemia basal ligeramente superior a la respuesta con la $\mathrm{BR}(\mathrm{t}=2.7$, $\mathrm{p}=0.01$ ), lo cual puede deberse a la dieta de los participantes un día antes de la prueba, a quienes se les solicitó mantener una dieta baja en carbohidratos, más no se les proporcionó una dieta que les aportará la misma cantidad de calorías y de carbohidratos lo que es un factor predisponente que puede alterar la glicemia basal (Parada \& Rozowski, 2008). Los resultados indicaron que la bebida con harina de semilla de ramón (BR) no alteró la respuesta glicémica de los participantes, considerando los criterios diagnósticos de la Asociación Americana de Diabetes (ADA, por sus siglas en inglés) y su condición de normoglicemia a los 120 min (ADA, 2018), de la misma forma que la bebida control (BC).
En los diferentes tiempos de respuesta no se presentó diferencia entre BC y BR, sin embargo, se observó que la respuesta del grupo BR disminuyó ligeramente en $5.9 \%$ a los $60 \min$ y a $3.7 \%$ a los $120 \mathrm{~min}$ en comparación con la BC.

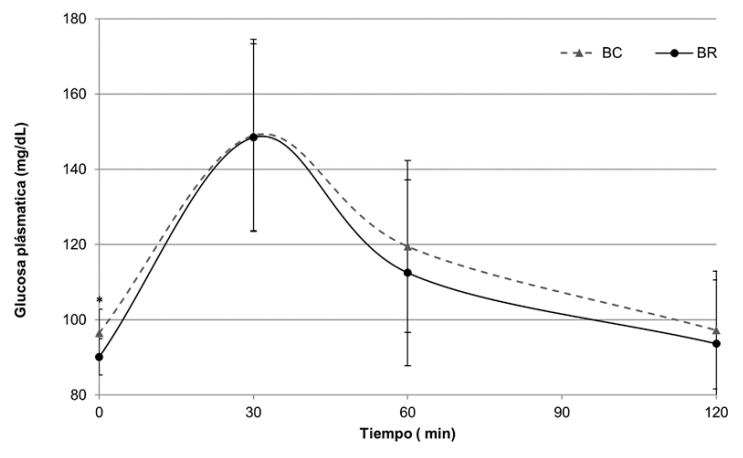

Figura 3. Curva de respuesta glicémica a la bebida adicionada con harina de semilla de Brosimum alicastrum Sw. Valores medios \pm DE. BC -bebida control, BR -bebida con harina de semilla de ramón. *Diferencia significativa a $p<0.05$.

Solamente se encontró un estudio que reporta un efecto hipoglucemiante en una bebida con harina de semilla de ramón, mostrando un decremento de la respuesta glicémica de $18.3 \%$.Esta diferencia puede relacionarse con el contenido de harina de semilla de ramón en una bebida tipo atole, la cual contenía 70.0 $\mathrm{g}$ de harina de semilla de ramón (23.3\%) en $300 \mathrm{~mL}$ de agua (Arévalo \& Bressani, 2013), lo cual fue 5 veces más que la cantidad de harina de semilla de ramón proporcionada en la bebida de este estudio $(14.0 \mathrm{~g} / 250 \mathrm{~mL})$. De acuerdo con Arévalo y Bressani, este efecto glicémico se atribuye a tres factores: uno el contenido de fibra dietética, el bajo índice glicémico de la bebida tipo atole $(<55 \%)$ y al bajo contenido de lípidos que presenta la harina de semilla de Brosimum alicastrum Sw. Considerando particularmente la fibra dietética, la diferencia de fibra $(1.8 \mathrm{~g} / 250 \mathrm{~mL})$ es 5 veces menos en la bebida de ramón del presente estudio en comparación con la bebida tipo atole $(9 \mathrm{~g} / 250 \mathrm{~mL})$ (Arévalo \& Bressani, 2013), esto podría explicar la diferencia en la respuesta glicémica entre ambos estudios. No obstante, el aumento de harina de semilla de ramón provoca alta viscosidad en la bebida, propiedad que no fue del agrado del consumidor cuando se probó la formulación inicial con harina de semilla ramón en $10.3 \%$. Esto confirma una vez más la importancia de considerar las propiedades organolépticas de los alimentos en paralelo a su funcionalidad. 
Por otra parte, el consumo de dietas especiales generalmente está relacionado para el tratamiento de enfermedades como alergias alimentarias, diabetes, intolerancia a la lactosa, enfermedad celíaca, entre otras. Sin embargo, también hay dietas especiales debido al estilo de vida, para bajar de peso, deportivas o culturales. Las dietas especiales han mostrado una tendencia creciente en los últimos años, siendo las dietas más frecuentes entre jóvenes: las vegetarianas y por intolerancia a la lactosa. No obstante, la dieta libre de gluten, para tratar la enfermedad celíaca, representa en un hoy en día un próspero mercado en el campo de los alimentos y las bebidas (Miranda, Lasa, Bustamante, Churruca, \& Simon, 2014). Este tipo de dieta selecciona alimentos que no solamente estén libres de gluten, sino que sean nutricionalmente adecuados. Diferentes cereales, granos, semillas legumbres y nueces pueden reemplazar al gluten (el amaranto, la quinoa, el mijo, el sorgo, el lino y los garbanzos); todos ellos pueden mejorar el sabor y calidad nutricional de la dieta, sin embargo, se usan con poca frecuencia, debido en parte al costo y su disponibilidad (Bascuñán, Vespa, \& Araya, 2017). En este sentido, la bebida adicionada con harina de semilla de Brosimum aliscatrum Sw. constituye una alternativa para una dieta libre de gluten, ya que utiliza una semilla rica en nutrientes y con poco uso en la actualidad. Los productos elaborados con esta semilla pueden beneficiarse por su aporte en macro y micronutrientes, a diferencia de los productos sin gluten, que actualmente se caracterizan por utilizar pocos ingredientes y no están fortificados como los equivalentes que contienen trigo. Algunas propuestas de alimentos procesados a partir de amaranto, quinua y trigo sarraceno han mostrado niveles más altos de proteínas, grasa, fibra y minerales en comparación con productos basados en granos tradicionales como el arroz y el maíz (Bascuñán et al., 2017). En este estudio, la harina de semilla de ramón representó una fuente de nutrientes para diseñar una bebida nutritiva que puede incorporarse a este tipo de régimen dietético y al estar libre de lactosa, puede ser una alternativa ante la creciente demanda de mercado por intolerancia a este azúcar. Por ejemplo, el consumo de bebidas basadas en plantas se ha incrementado (19\%), estas bebidas principalmente son elaboradas a partir de soya, arroz, coco, avena o quinua, las cuales son nutrimentalmente más pobres que la leche de vaca, particularmente con menor contenido de proteína (excepto la de soya) o con pobre calidad proteica (PDCAAS), como es el caso de la bebida de almendra. El bajo contenido de aminoácidos esenciales en bebidas como la de coco, arroz, quinua, almendra o avena (aminoácidos sulfurados) (Sousa \& Kopf-Bolanz, 2017) es particularmente importante a considerar, si se desea tener una dieta balanceada. En contraparte, la harina de semilla de ramón proporciona nueve aminoácidos esenciales (histidina, isoleucina, lisina, metionina, fenilalanina, treonina, triptófano y valina (Carter, 2015), además de fibra y calcio (Larqué-Saavedra, 2014a), que no contienen algunas bebidas elaboradas a partir de plantas o son muy pobres en estos nutrientes, por lo que deben ser fortificadas (Sousa \& Kopf-Bolanz, 2017). Finalmente, la bebida de ramón tiene un sabor a café capuchino y su consistencia y aspecto son similares a este producto, con la diferencia de que no contiene cafeína como otras bebidas (café, té, refresco o bebidas energizantes) consumidas comúnmente en la dieta. Esto representa una ventaja para aquellos individuos con sensibilidad a la cafeína o bien que su consumo en exceso (>400 mg/día) provoca efectos adversos como ansiedad, depresión, dificultad para dormir, temblores, náuseas, entre otros) (Wolde, 2014). Actualmente las directrices dietéticas basadas en alimentos (FBDG, por sus siglas en inglés) proporcionan una visión desfavorable de las bebidas con cafeína, especialmente por sus propiedades diuréticas, psicoestimulantes e inhibidora de nutrientes (Reyes \& Cornelis, 2018), lo que hace de la bebida de ramón un alimento que puede ser incluido en la dieta habitual. La semilla de ramón representa una alternativa en el diseño de nuevos alimentos en contraste con otros granos o semillas de uso convencional.

\section{Conclusiones}

La bebida adicionada con harina de semilla de Brosimum alicastrum Sw. (ramón), formulada en el presente estudio, fue sensorialmente aceptada por el consumidor, con un aporte nutrimental de interés, particularmente en su contenido de proteína, fibra dietética, libre de gluten, lactosa y cafeína, comparativamente con bebidas de este tipo, y su consumo mostró una respuesta glicémica posprandial normal. Por lo anterior, este tipo de bebida puede considerarse como una propuesta atractiva de consumo regular entre la población y particularmente una alternativa para jóvenes adultos 
con requerimientos especiales de alimentación por intolerancia a la lactosa, gluten o cafeína.

\section{Agradecimientos}

Los autores agradecen al Centro de Investigación Científica de Yucatán (CICY) por la donación de la harina de semilla de Brosimum alicastrum Sw. para realizar este estudio.

\section{Bibliografía}

Acosta, A., \& Martínez-Ruiz, N. (2017). Desarrollo de una bebida nutritiva y sensorialmente agradable como suplemento en el desayuno de niños escolares. Revista Española de Nutrición Comunitaria, 23(1), 1-9.

ADA, (American Diabetes Association). (2018). Classification and diagnosis of diabetes: Standards of Medical Care in Diabetes. Diabetes Care, 41(Suppl. 1), S13-S27.

AOAC. (2000). Official Methods of Analysis (17th ed.). USA: AOAC International.

Arévalo, A., \& Bressani, R. (2013). Respuesta glicémica de la semilla de Ramón (Brosimum alicastrum) en mujeres de 16 a 25 años de edad, residentes de la ciudad capital, Guatemala. Revista 25 de La Universidad Del Valle de Guatemala, 66-71.

Barrance, A., Beer, J., Boshier, D., Chamberlain, J., Cordero, J., Detlefsen, G., ... Pennington, T. (2003). Arboles de Centroamerica: un Manual para Extensionistas (Trees of Central America: a Manual for Extentionists). (J. Cordero \& D. Boshier, Eds.). Turrialba, Costa Rica: OFI/CATIE.

Bascuñán, K., Vespa, M., \& Araya, M. (2017). Celiac disease : understanding the gluten - free diet. European Journal of Nutrition, 56(2), 449-459.

Bedolla, B., Dueñas, C., Esquivel, I., Favela, T., Guerrero, R., Mendoza, E., ... Trujillo, M. (2004). Introducción a la tecnología de alimentos (2da.). México: Limusa.

Carter, C. T. (2015). Chemical and Functional Properties of Brosimum Alicastrum Seed Powder ( Maya Nut, Ramón Nut ). Clemson University, USA.

CONAFOR-FAO. (2012). The State of the Worl's Forest Genetic Resources. Country Report Mexico. Rome, Italy: FAO.

Connor, W. E. (2000). Importance of $\mathrm{n}-3$ fatty acids in health and disease. American Journal of Clinical Nutrition, 71(Suppl), 171S-5S.
Davidsohn, I., \& Bernard, J. (1983). Diágnóstico Clínico por el Laboratorio (6a.). Barcelona, España: Salvat Editores S.A.

Gutiérrez, A. (2017, September). El mujú (Brosimum alicastrum), una alternativa en la estrategia contra la pobreza alimentaria en Chiapas. Revista Más Agro, $28-31$.

Larqué-Saavedra, A. (2014a). ). Brosimum alicastrum. Ramón. El sector forestal en apoyo a la cruzada contra el hambre. Gaceta, 6(49), 6-35.

Larqué-Saavedra, A. (2014b). Brosimum alicastrum. Ramón. Gaceta, 6(49), 6-33.

Larqué-Saavedra, A. (2014c). El sector forestal en apoyo a la cruzada contra el hambre. Forestal XXI, 17(2), 1112.

Lawless, H., \& Heymann, H. (2010). Sensory Evaluation of Food (2nd ed.). USA: Springer.

México 3M. (2017). Recuento de Microorganismos (3M Recuento de Aerobios AC, Coliformes y Hongos y Levaduras). México DF.

Miranda, J., Lasa, A., Bustamante, M., Churruca, I., \& Simon, E. (2014). Nutritional Differences Between a Gluten-free Diet and a Diet Containing Equivalent Products with Gluten. Plant Foods for Human Nutrition, 69, 182-187.

Molina-Rosell, C. (2013). Alimentos sin gluten derivados de cereales. In L. Rodrigo \& A. Peña (Eds.), Enfermedad celíaca y sensibilidad al gluten no celíaca (pp. 447-461). Bercelona, España: OmniaScience.

Moreira, V., \& López San Román, A. (2006). Intolerancia a la lactosa. Revista Española de Enfermedades Digestivas, 98(2), 143.

Moscoso, F., \& Quera, R. (2015). Enfermedad Celiaca: Revisión. Rev. Med. Clin. CONDES, 26(5), 613-627.

NAS. (1975). Underexploited tropical plants with promising economic value. Washington, DC, USA.

Ozer, H. K. (2017). Phenolic compositions and antioxidant activities of Maya nut (Brosimum alicastrum): Comparison with commercial nuts. International Journal of Food Properties, 20(11), 2772-2781.

Parada, J., \& Rozowski, J. (2008). Relación entre la respuesta glicémica del almidón y su estado microestructural. Revista Chilena de Nutrición, 35(2), 84-92. 
Pérez, S., Baldwin, P., \& Gallant, D. (2009). Structural features os starch granules I. In J. BeMiller \& R. Whistler (Eds.), Starch:Chemistry and Technology (3rd ed., pp. 149-188). USA: Academic Press.

Peters, C. M., \& Pardo-Tejeda, E. (1982). Brosimum alicastrum (Moraceae): uses and potential in Mexico. Economic Botany, 36(2), 166-175.

Reyes, C., \& Cornelis, M. (2018). Caffeine in the Diet: Country-Level Consumption and Guidelines. Nutrients, 10(1772), 1-34.

Richards, G., \& Smith, A. (2015). Caffeine consumption and self-assessed stress, anxiety, and depression in secondary school children. Journal of Psychopharmacology, 29(12), 1236-1247.

Rosado, J. L. (2016). Intolerancia a la lactosa. Gaceta Médica de México, 152(Sppl 1), 67-73.

SE, (Secretaría de Economía). (2012). NOM-183-SCFI2012. Producto lácteo y producto lácteo combinadoDenominaciones, especificaciones fisicoquímicas, información comercial y métodos de prueba. México D.F.

Sousa, A., \& Kopf-Bolanz, K. (2017). Nutritional Implications of an Increasing Consumption of NonDairy Plant-Based Beverages Instead of Cow' s Milk in Switzerland Advances in Dairy Research. Advances in Dairy Research, 5(4), 1-7.

SS, (Secretaria de Salud). (2002). NOM-184-SSA1-2002. Productos y servicios. Leche, fórmula láctea y producto lácteo combinado. Especificaciones sanitarias. México D.F.

SSA, (Secretaría de Salubridad y Asistencia). (1978). NMX-F-317-S-1978. Determinación de pH en alimentos. México D.F.

SSA, (Secretaría de Salubridad y Asistencia). (1979). NMX-F-090-S-1978. Determinación de pH en alimentos. México D.F.

SSA, (Secretaría de Salubridad y Asistencia). (1981). NMX-F-360-S-1981. Alimentos para humanos. Determinación de cloruros como cloruro de sodio (Método de Volhard). México D.F.

Sundaram, J. K., Rawal, V., \& Clarck, M. T. (2015). Ending malnutrition from commitment to action. (FAO, Ed.) (Vol. 53). India: FAO.

Suri, S., Kumar, V., Prasad, R., Tanwar, B., Goyal, A., Kaur, S., ... Singh, D. (2019). Journal of Nutrition \&
Intermediary Metabolism Considerations for development of lactose-free food. Journal of Nutrition \& Intermediary Metabolism, 15(November 2018), 2734.

Tirado, D. F., Montero, P. M., \& Acevedo, D. (2015). Aceptabilidad Sensorial y Calidad Microbiológica de Bebidas a Base de Arroz y Plasma Bovino y Porcino. Informacion Tecnologica, 26(6), 45-54.

Tokpunar, H. K. (2010). Chemical composition and antioxidant properties of maya nut (Brosimum alicastrum). Clemson University, USA.

USDA. (2017). National Nutrient database for Standard Reference. Agricultural Research Service. Retrieved from https://ndb.nal.usda.gov/ndb/foods/

Wolde, T. (2014). Effects of caffeine on health and nutrition: A Review. Food Science and Quality Management, 30, 59-66.

Yang, A., Palmer, A., \& de Wit, H. (2010). Genetics of caffeine consumption and responses to caffeine. Journal of Psychopharmacology, 211(3), 245-257. 